\title{
Biomimetic sensing layer based on electrospun conductive polymer webs ${ }^{\text {th }}$
}

\author{
E. Zampetti ${ }^{a}$, S. Pantalei ${ }^{a}$, S. Scalese ${ }^{b}$, A. Bearzotti ${ }^{a}$, F. De Cesare ${ }^{a, c}$, C. Spinella ${ }^{b}$, A. Macagnano ${ }^{a, *}$ \\ a Institute for Microelectronics and Microsystems-Rome (IMM-Rome), Via Fosso del Cavaliere 100, 00133 Rome, Italy \\ ${ }^{\mathrm{b}}$ Institute for Microelectronics and Microsystems-Catania (IMM-Catania), VII Strada 5, 95121 Catania, Italy \\ ${ }^{c}$ Agrobiology and Agrochemistry Department (DABAC), University of Tuscia, Via S. Camillo de Lellis, 01100 Viterbo, Italy
}

\section{A R T I C L E I N F O}

\section{Article history:}

Received 27 July 2010

Received in revised form

30 September 2010

Accepted 19 October 2010

Available online 27 October 2010

\section{Keywords:}

Bioinspired systems

Nanofibres

PANi blends

Electrospinning

Electronic nose

\begin{abstract}
A B S T R A C T
The aim of the present study is to combine a bio-inspired nanofibrous artificial epithelium to the electronic nose (e-nose) principles. The sensing device set up was an electronic nose consisting of an array of 9 microchemoresistors $(\mathrm{Cr}-\mathrm{Au}, 3 \times 3)$ coated with electrospun nanofibrous structures. These were comprised of doped polyemeraldine base blended with 3 different polymers: polyethylene oxide, polyvinilpyrrolidone and polystyrene, which acted as carriers for the conducting polymer and were the major responsible of the features of each fibrous overlay (electrical parameters, selectivity and sensitivity ranges). The two sensing strategies here adopted and compared consisted in the use of 2 different textural coatings: a single- and a double-overlay, where the double-overlay resulting from overdeposition of 2 different polymer blends. Such e-nose included a plurality of nanofibres whose electrical parameters were at the same time depending on each polymer exposure to analytes $\left(\mathrm{NO}_{2}, \mathrm{NH}_{3}\right)$ and on the spatial distribution of the interlacing fibres. The morphology of the coating arrangements of this novel e-nose was investigated by scanning electron microscopy (SEM) and its sensor responses were processed by multicomponent data analyses (PCA and PLS) reporting encouraging results for detection and recognition of analytes at ppb levels.
\end{abstract}

(c) 2010 Elsevier B.V. All rights reserved.

\section{Introduction}

There is considerable interest in developing sensors that act on the analogy of the mammalian olfactory system. They can be designed to mime natural materials, structures, strategies etc. The recent massive development of micro- and nanotechnologies results to be a suitable support to create both materials and tools more and more similar to the natural arrangement (Beccherelli et al., 2010; Macagnano et al., 2008). Actually, the idea to develop an artificial olfaction technology had its beginnings in 1982, with the invention of the first "electronic nose" (Persaud and Dodd, 1982). The starting aims were mainly devoted to create novel systems to complement techniques of analytical chemistry (Gardner and Bartlett, 1991) to classify gas mixtures and odours, subsequently, such an electronic system has been suitably designed and made up for various applications in several fields, as security (Arshak et al., 2006), environment (De Cesare et al., 2008), health (Pavlou et al., 2002; D’Amico et al., 2006; Di Natale et al., 2003), foodstuff (Olafsdottir et al., 2004), cultural heritage (Canhoto et al., 2004), etc. In recent years, considerable progress has been made in understanding how the mammalian olfactory system (Nef, 1998; Satir and Christensen, 2007) uses to detect the odours and encode sen-

\footnotetext{
is The paper was presented at the World Congress on Biosensors 2010.

* Corresponding author. Tel.: +39 0649934539; fax: +39 0649934066.

E-mail address: antonella.macagnano@artov.imm.cnr.it (A. Macagnano).
}

sory information (Goepel, 1998; Bathellier et al., 2008). The focal point is lying in the combinatorial code of the natural system which can be supposed to be based on the idea that one receptor responds to many analytes and many receptors respond to any given analyte (Goepel et al., 1998; Tzong-Zeng, 1999; Pearce et al., 2003). Thus, an artificial sensor array has to be designed comprised of broad selectivity and sensitivity to different chemical classes. The sensor array strategy forecasts a collection of sensors containing chemical diversity as much as possible to respond to the largest possible cross-section of analytes. Each artificial receptor in a sensor array has to be designed with appropriate chemical features (functional groups, metal-cores, molecular cavities, etc.) such as a suitable interacting framework. At this regard, the olfactory epithelium surface consists of tiny hair-like cilia protruding from the olfactory receptor cell's dendrite into the mucus, bringing about a remarkable increase of the small surface area (about $2.5 \mathrm{~cm}^{2}$ per nostril) (Satir and Christensen, 2007). Obviously, the increase of the interacting surface improves the surrounding chemical detection. Thus, nanofibrous interacting layers are expected to enhance the available sensing surface area, providing an increase in sensitivity and capture efficiency too. In the present work both electronic nose and bio-inspired nanofibrous artificial epithelium principles have been combined. The nanofibrous polymeric structures have been fabricated adopting electrospinning deposition technique (Reneker and Chun, 1996; Wang et al., 2009), which is a versatile process by which polymer nanofibres with diameters ranging from few 
nanometres to several micrometers can be produced and deposited at once, using an electrostatic force to drive the jet of polymer solution. These structures can have surface areas approximately one or two orders of magnitude higher than those found in continuous films. Furthermore, such a structure may be a better dynamic system than a compact film, allowing a faster adsorption process and avoiding some bulk effects (i.e., long diffusion-desorption time, analyte entrapments, etc.). A suitable array of 9 chemoresistors fabricated on a silicon wafer has been coated with different electrospun nanofibres of conductive polymers (CPs) (Bai and Shi, 2007) according to a defined topological layout. Since polyaniline is one of the most interesting CPs for its conductive features (when doped), its thermal stability and its sensing performances (Kukla et al., 1996; Nicolas-Debarnot and Poncin-Epaillard, 2003; Virji et al., 2004), it has been selected and investigated as crucial in the construction of the sensor array. Thus, at first, blends of polyaniline and insulating host polymers (Norris et al., 2000; Bishop and Gouma, 2005; Aussawasathien et al., 2005) have been planned, prepared and studied to verify, contemporarily, the combination of electrical conductivity of the conductive polymer and the physical properties of the host polymer. Secondly, a combination of overlaid and double-overlaid areas linked by interconnections of different fibres have been created and investigated as potential sensors. The selected overdepositions of couples of fibrous polymers, with their interconnections, introduced broader and overlapping sensitivities to different analytes, making each sensor a desirable sensing element of an artificial olfactory system. Such an Interdigitated sensor array (ISA), included a plurality of nanofibres whose electrical parameters were contemporarily depending on each polymer exposure to analytes and on the spatial distribution of the interlaced fibres. The choice of the hosting polymer depends on the stability of the polymer, gas/vapour transport ability and affinity to the CP: it operates dually as an absorbent for target analytes and a filter for unwanted interferents. The selected hosting polymer carriers (respectively polyvinilpyrrolidone, PVP, polyethylene oxide, PEO and polystyrene, PS) modifying remarkably the typology of the interacting surface (diameter and length of the fibres, roughness, porosity, presence of beads and grains, no woven and/or branched framework) in addition to the different affinity to the tested analytes, have allowed to the ISA getting a wider dynamic range of sensitivities. The morphology of the coating arrangements of this novel e-nose has been investigated by scanning electron microscopy (SEM) and its sensor responses to two gases, at ppb levels, were processed by multicomponent data analyses: principal component analysis (PCA) and partial least square (PLS) (Massart et al., 1998; Hierlemann et al., 1996). Furthermore, sensors were tested under increasing water vapour concentrations in order to estimate the potentials of the designed polymeric array (swelling, morphology changes, reliability) to humidity interference.

\section{Materials and methods}

\subsection{Polymers and reagents}

Polyaniline emeraldine base (PANi, MW=10,000), 10-camphorsulfonic acid (CSA), polyethylene oxide (PEO, MW =100,000), polyvinilpyrrolidone (PVP, MW $=1,300,000$ ) and polystyrene (PS, $\mathrm{MW}=192,000$ ) were purchased from Aldrich and used without further purification. Solvents were purchased as follows: chloroform $\left(\mathrm{CHCl}_{3}, 99.8 \%\right.$ J.T. Baker), ethanol (EtOH, anhydrous, Carlo Erba Reagents) and tetrahydrofuran (THF, Sigma Aldrich).

\subsubsection{PANi-PS blend preparation}

$400 \mathrm{mg}$ of PANi was doped with $641 \mathrm{mg}$ of CSA and dissolved in $10 \mathrm{ml}$ of $\mathrm{CHCl}_{3} /$ THF mixture $(9: 1, \mathrm{v} / \mathrm{v})$ for a period of $8 \mathrm{~h}$ with mag- netic stirring at room temperature. The deep-green solution was filtered $(0.5 \mu \mathrm{m}$ polytetrafluoroethylene-(PTFE) filter, Millipore) to get a homogeneous solution and $750 \mathrm{mg}$ of PS was added to the solution and stirred for an additional $6 \mathrm{~h}$.

\subsubsection{PANi-PEO and PANi-PVP blends preparation}

$800 \mathrm{mg}$ of PANi was doped with $1.282 \mathrm{~g}$ of CSA and dissolved in $20 \mathrm{ml}$ of $\mathrm{CHCl}_{3} / \mathrm{EtOH}$ mixture $(9: 1, \mathrm{v} / \mathrm{v})$ for $8 \mathrm{~h}$ with magnetic stirring at room temperature. The resulting deep-green solution, filtered throughout a $0.5 \mu \mathrm{m}$ PTFE filter (Millipore) and split into two $10 \mathrm{ml}$ solutions, was added respectively with $602 \mathrm{mg}$ of PVP and $250 \mathrm{mg}$ of PEO and stirred for an additional $6 \mathrm{~h}$.

\subsection{Interdigitated sensor array layout}

The base transducer element used in the present work was an interdigitated electrode with 40 pairs of electrodes and gaps of $20 \mu \mathrm{m}$ (Janata and Josowicz, 2003). Each electrode was long $5620 \mu \mathrm{m}$. A suitable array of 9 interdigitated electrodes was fabricated on a 4 inches oxidized silicon wafer, through a standard photolithographic process with lift-off technique (Wolf and Tauber, 2000). The metallization layer was made by evaporation of a chromium-gold film (150 nm thick).

A dielectric layer ( $2 \mu \mathrm{m}$ of negative tone SU8 photoresist, permanently cross-linked) was uniformly deposited all over the device and photolithographically opened in correspondence of the transducers allowing both the electrospinning deposition just over the transducers and the protection of internal metal connections. The organic deposits due to micro-processing were removed by ultrasonic treatment in acetone for $2 \mathrm{~min}$ and in 2-propanol for $1 \mathrm{~min}$, respectively, then the electrodes were dried under a filtered nitrogen stream.

\subsection{Electrospinning, deposition and measurement setup}

In a typical electrospinning setup an electrical potential is applied between a droplet of a polymer solution held at the end of a capillary tube and a grounded target: when the electric field that is applied overcomes the surface tension a charged jet of polymer solution is ejected. In the reported study the depositions were carried out in a homemade clean box comprised of temperature and humidity sensors $\left(24^{\circ} \mathrm{C}, 30 \% \mathrm{RH}\right)$. The syringe containing the polymer blend was placed horizontally on the syringe pump (KDS-200, KD Scientific, feed rate $1 \mathrm{ml} / \mathrm{h}$ ) with the tip-collector distance of about $5 \mathrm{~cm}$. The collector consisted of a rotating conductive cylin$\operatorname{der}$ ( $2000 \mathrm{rpm}, \varnothing=45 \mathrm{~mm}$ ), housing the ISA, able to increase the quantity of fibres assembled per unit of time. The steel needle with flat tip $\left(\varnothing_{\text {in }}=300 \mu \mathrm{m}\right)$ was connected to the high voltage generator $(10 \mathrm{kV} \mathrm{DC})$ and the deposition time was fixed at $300 \mathrm{~s}$ for all the polymer materials. The ISA consisted of 9 interdigitated electrodes (IDEs) and 3 of them were coated with different electrospun nanofibres of a single polymer blend (PANi-PEO, PANi-PS and PANiPVP, respectively) while the other 6 electrodes were coated with combinations of 2 polymer blends, creating overlaid (one fibrous polymer) and double-overlaid (two different fibrous polymers) areas. After each deposition, the wafer was housed in an incubator at $40^{\circ} \mathrm{C}$ for $5 \mathrm{~h}$, under nitrogen flow. The materials deposited on ISA were investigated by scanning electron microscopy, using a high resolution FE-SEM Zeiss Supra 35, with an electron beam energy ranging from 0.1 to $30 \mathrm{kV}$, a Schottky field emitter and an in-lens SE detector. In this work, low energy $(<3 \mathrm{keV})$ electron beam was used in order to avoid deterioration of the polymer fibres. PANiPEO layer showed a very good reproducibility in coating, as well as PANi-PS. It was characterized by very tiny fibres (ranging between $100 \mathrm{~nm}$ and $400 \mathrm{~nm}$ ), with irregular and grainy surface. More soft and gauze-like appeared PANi-PVP layer, with a nonwoven elec- 


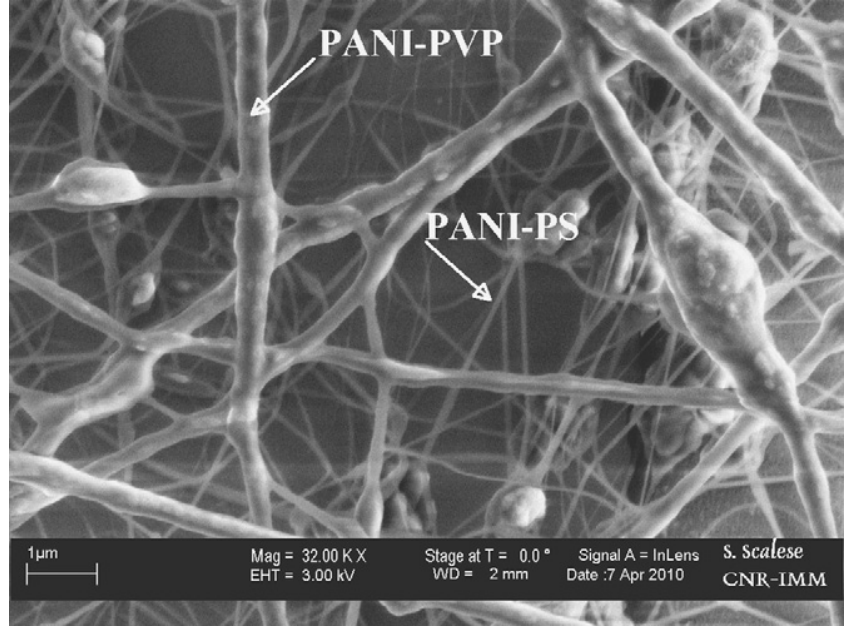

Fig. 1. SEM micrograph PANi-PS/PANi-PVP (under- and overlayer, respectively).

trospun mat and branched fibres. Fibres were more irregular in diameter (50-600 $\mathrm{nm}$ ) and shape (rough) than PANi-PS. PANi-PS fibres were smooth and fine $(<300 \mathrm{~nm})$, and the scaffold was comprised of homogeneous fabric. A straight comparison between two typologies of fibres can be observed in Fig. 1 where a layer of PANi-PS is coated with PANi-PVP. It is possible to observe the differences, in terms of fibre dimensions, coverage and web morphology, between the two different overlaid polymers. All the resulting fabrics were highly porous, having a large interconnected void volume, and possessing high surface-to-volume ratios. The polymer fibres covered both the electrodes and the gap between them assuring the electrical continuity.

The sensor device was placed into the measurement chamber and the ISA pads were electrically connected to an ammeter (Keithley 6517A Electrometer), a fast electromechanical multiplexer (MUX 9:1) and a microcontroller unit. The resulting error in the measured current was about $\pm 100 \mathrm{pA}$. The multiplexing time was $50 \mathrm{~ms}$, to obtain a measure of the whole ISA device in about $0.5 \mathrm{~s}$. Temperature and relative humidity values were measured by an analog to digital converter (ADC) channel of the microcontroller unit. The electrical features of the fabricated chemo-resistors were investigated by measuring current-voltage values, ranging between $-1 \mathrm{~V}$ and $+1 \mathrm{~V}$ to avoid degradation caused by self-heating. Dynamic measurements were carried out at room temperature using a 4-channel MKS 247 readout, driving four MKS mass flow controllers where $\mathrm{N}_{2}$, the gas carrier (Praxair-Rivoira, Italy), was blended to increasing concentration respectively of $\mathrm{NH}_{3}$ and $\mathrm{NO}_{2}$ by cylinders at known concentrations ( $5 \mathrm{ppm} / \mathrm{N}_{2}$, Praxair-Rivoira, Italy) and to controlled percentages of vapours water by bubbling. The total gas flow of $200 \mathrm{sccm}$ (standard cubic centimetres per minute) passed through both the glass mixing chamber $\left(\sim 5 \mathrm{~cm}^{3}\right)$ and the steel-teflon measurement chamber housing both the IDE array and a temperature/humidity sensor (Honeywell HIH 3602C). The response to each concentration was measured five times in five repeated exposures (length of about $90 \mathrm{~s}$ and $180 \mathrm{~s}$ respectively for $\mathrm{NH}_{3}$ and $\mathrm{NO}_{2}$ ). Each measurement was carried out after the complete recovery of the starting current when pure $\mathrm{N}_{2}$ was flowed.

\subsection{Data analysis}

The data set resulting from sensor readouts consisted of the responses of all the 9 sensors to different concentrations of both $\mathrm{NH}_{3}$ and $\mathrm{NO}_{2}$ with nitrogen (from $125 \mathrm{ppb}$ to $5000 \mathrm{ppb}$ ) and to the variation of relative humidity in nitrogen (from about 10\% to 30\%) with nitrogen.
The data set was analyzed using standard multivariate data analysis techniques through commercial software MATLAB 7.1 (The MathWorks, Inc.) and PLS ToolBox 5.8 (Eigenvector Research Inc.).

At first, the PCA was carried out, as an unsupervised classification technique, to depict how the sensors responses were correlated one to each other. Biplot of loadings on PCA was performed to point out how sensors responded to the different analytes and how they together contributed to the classification of the chemical compounds.

Secondly, the PLS-Discriminant Analysis (PLS-DA) was performed on the data set to evaluate the potential of the sensor array to qualitatively predict which class of chemicals an unknown measurement belongs to.

Finally, a regression model was built through Partial least square (PLS) technique to quantitatively estimate the analyte concentration on the base of a certain sensor array response.

PLS-DA and PLS regression are supervised techniques which involve a validation process in order to assess how correctly the generated models recognize independent, unknown data. This process can involve the leave-one-out cross validation method (LOOCV) to assess the reliability of the model. This procedure consists in removing one measure from the original data set and using it as a validating data, while the remaining data set is used to assess the model. This process is repeated until every measure is used once as a validating data. The output of the overall validating method resides in the average of the single validations. These multivariate analyses are simple and powerful techniques commonly used in electronic nose applications (Hierlemann et al., 1996; Pearce et al. 2003).

\section{Results and discussion}

\subsection{Electrical signals measurements}

The selected sensor array produced sensors with comparable conductivities and good quality of fibres. The linear shape of the whole current-voltage curves along the selected voltage range $(-1 \mathrm{~V}$ to $+1 \mathrm{~V})$ indicated a constant resistance value not depending on the current flowing into the device. It suggested a good adherence of the whole polymers to the electrode and that the gold contact did not introduce apparent effects of nonlinearity (Schottky barrier) (Hao et al., 2003; Li et al., 2004). In fact the electrical resistance of the sensor elements is related to the individual fibres resistance (due to dimension and shape), fibre density and the electrode coverage (i.e., texture and adhesion) as well as to the $\mathrm{CP} / \mathrm{P}_{\text {host }}$ ratio $(\mathrm{w} / \mathrm{w})$ : PANi amount in PVP and PS are similar (0.66 and 0.53, respectively) while in PEO is more than double (1.6). On the other hand, the resulting conductive properties are depending also on parameters such as both the affinity with the hosting polymer, the adopted solvent and the deposition setting. The resulting electrical resistance mean values measured for each layer over electrodes, were $3.88 \pm 1.19 \mathrm{k} \Omega$, $7.05 \pm 2.7 \mathrm{k} \Omega$ and $7.52 \pm 3.09 \mathrm{k} \Omega$ respectively for PANi-PEO, PANiPS and PANi-PVP. These low levels of resistance, ensured low values of thermal electronic noise, thus increasing the performances of the sensory system. The Ohmic behaviour was still maintained after the over-deposition, showing a substantial decrease in resistance for each sensor. Such a result let hypothesize a structure comparable to two resistors in parallel, since the whole resistance values decreased as a consequence of the over-deposition. Indeed, the resistance of the overlaid sensors with the same polymer decreased too $\left(R_{\mathrm{PEO} / \mathrm{PEO}}=1.34 \pm 0.38 \mathrm{k} \Omega, R_{\mathrm{PVP} / \mathrm{PVP}}=2.03 \pm 0.71 \mathrm{k} \Omega\right.$ and $R_{\mathrm{PS} / \mathrm{PS}}=2.15 \pm 0.7 \mathrm{k} \Omega$ ). The sensor array getting the lowest resistance values was selected here for gas detection. In order to evaluate the ISA sensitivity to $\mathrm{NO}_{2}, \mathrm{NH}_{3}$ and relative humidity vari- 


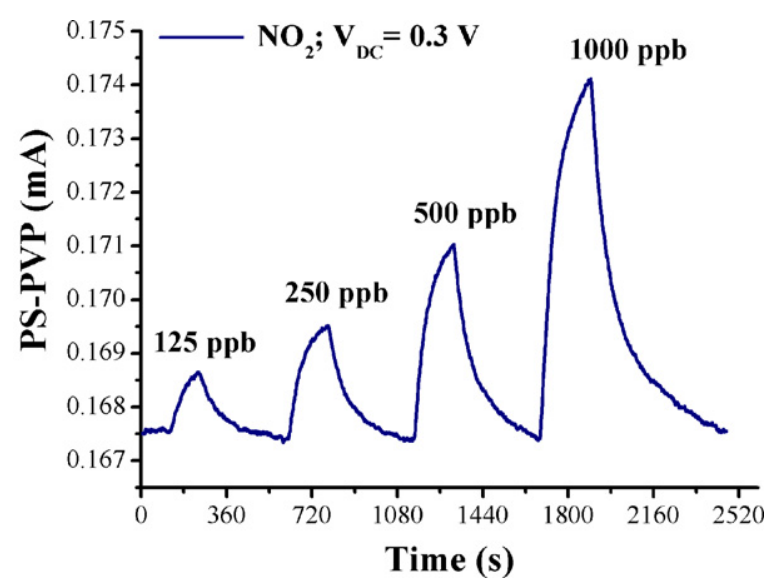

Fig. 2. Dynamic response of PANi-PS/PANi-PVP sensor in the presence of increasing concentrations of $\mathrm{NO}_{2}$ supplied through pulses of $180 \mathrm{~s}$. The supply voltage $(\mathrm{V})$ was set at $0.3 \mathrm{~V}$.

ations, different pulses of increasing concentrations were provided to the device. The cyclic measurements of $\mathrm{NO}_{2}$ were supplied to the sensors when they were undergoing a thorough cleaning. Fig. 2 shows the dynamic response, in terms of current changes (I) of an ISA's element, i.e., the double layer PANi-PS/PANi-PVP (underand overlayer, respectively) when increasing concentrations of $\mathrm{NO}_{2}$ were flowed throughout the measurement chamber.

The high porosity of the sensors allowed getting rapid changes in current (fast responses) when the gas was respectively sorbed and desorbed. Furthermore, since each sensor of such a nanostructured device could detect, with different sensitivity, very low concentrations of both ammonia and nitrogen dioxide (up to ppb levels), it is possible to suppose potential employments of the proposed artificial nose as a diagnostic instrument, able to detect and reveal gases involved in metabolic diseases and released in traces from breath and skin (Turner et al., 2008). This assumption is supported by previous studies on the sensing features of a polyaniline-based sensor array (Macagnano et al., 2009). The sensor response magnitude as a function of gas concentration ( $\mathrm{ppb}$ ) (calculated as percentage of $\Delta I / I_{0}$, where $\Delta I$ is the current variation and $I_{0}$ is the current when pure $\mathrm{N}_{2}$ is flowed), was measured for both $\mathrm{NO}_{2}$ and $\mathrm{NH}_{3}$ and it is illustrated in Fig. 3(a,b).

$\mathrm{NO}_{2}$, in fact, is an electron withdrawing able to remove electrons from the aromatic rings of the conducting polymers causing the changes in resistance and work function of the sensing material. When this occur at a $p$-type $C P$, such as polyaniline, the doping level as well as the electric conductance of the conducting polymer is enhanced. However each sensor shows different response value to the same concentration, depicting in such a way, a series of 9 curves with different slopes. This effect could be due to both changeable percentage of polyaniline in the different polymer hosts and its distribution all along the hosting fibres (inside/outside the fibre surface, in grains, in pores, etc.). For a comprehension of the sensing results, the different gas sorption and diffusion mechanisms too, inside the various polymeric fibres have to be considered. In particular, PANi-PEO, PANi-PEO/PANi-PVP and PANi-PEO/PANi-PS show less sensitivity to $\mathrm{NO}_{2}$ compared both to PANi-PVP (the highest one) and PANi-PS and their respective combinations.

The response curves of the same sensors to $\mathrm{NH}_{3}$ (Fig. 3b) show a completely different behaviour. In fact, an increasing in current is reported for all the sensors up to $1500 \mathrm{ppb}$, a dramatic change of trend (parabolic shape, negative convex coefficient) is then depicted, showing decreasing values of current (resistance increasing). This effect is more evident for PANi-PVP and PANi-PEO (and their combinations) even if it starts at different concentrations of $\mathrm{NH}_{3}$. On the contrary, PANi-PS shows increasing current up to $3500 \mathrm{ppb}$, then a slight decreasing effect is reported. So apparently, PANi-PS may be the most sensitive to very low concentrations of ammonia. In effect, the resulting behaviour of each fibrous layer seems to confirm the presence of competitive processes interaction of the $\mathrm{NH}_{3}$ with the PANi as explained from Kukla et al. (1996) where deprotonation (increase in resistance) is in competition with a hydrogen bond causing a complete symmetrization of the chemical bonds (increase in conductivity). The different sorption of $\mathrm{NH}_{3}$ in the three hosting polymers seems to enhance, in a different way, the presence of these two opposite effects, usually unlikely to demonstrate because of the lowest sensitivity to ammonia of the common sensors. The resulting spread of sensitivity of all the sensor curves for $\mathrm{NH}_{3}$ and $\mathrm{NO}_{2}$ is however a desirable requirement for designing a sensor array. The polymers tested demonstrated sufficient crossselectivity to analyze each of the tested gas using the sensor array pattern (Albert et al., 2000).

Since water could be an interfering parameter for gas detection, overall when gas in traces should be detected, preliminary results about the behaviour of the sensor array to water vapours up to $30 \% \mathrm{RH}$ (in order to avoid any kind of sensor array poisoning) was recorded and depicted in Fig. 3(c). Simultaneous mechanisms of different interactions can occur when water vapours are sorbed in the PANi-polymeric fibre as swelling, acid-doping solvation, $\mathrm{H}$ bonding, electron withdrawing, physical entrapment due to the high porosity and roughness, etc. Higher responses in current to water seem to be reported overall by the combination of all the double layers. Probably where swelling is prevailing, the response curve, related to the current, has a slighter slope. PANi-PVP, a strongly hydrophilic polymer, indeed had the lowest responses in current, showing a parabolic shape (negative convex coefficient).

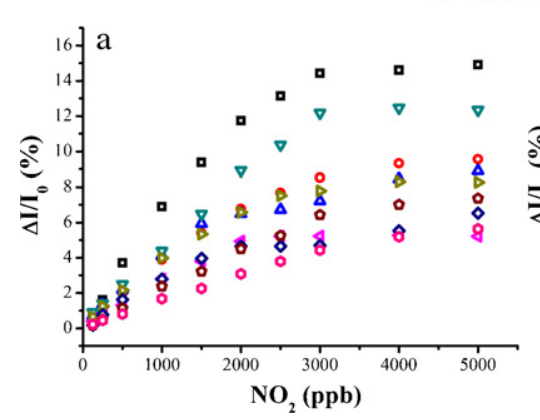

$\square$ PANi-PVP $\bigcirc$ PANi-PVP/PANi-PEO $\triangle$ PANi-PVP/PANi-PS

$\nabla$ PANi-PS $\triangleleft$ PANi-PS/PANi-PEO $\triangleright$ PANi-PS/PANi-PVP

$\diamond$ PANi-PEO $\triangle$ PANi-PEO/PANi-PS $\bigcirc$ PANi-PEO/PANi-PVP
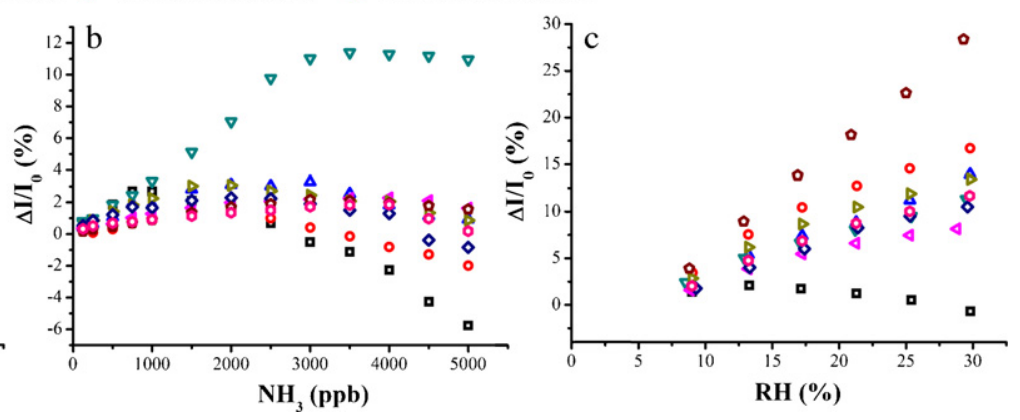

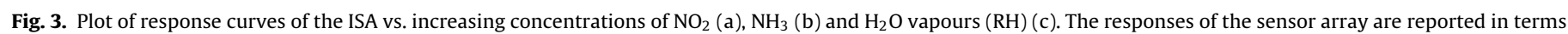
of per cent of relative current variations $\Delta I / I_{0}$, where $I_{0}$ was the measured current, for each sensor, in presence of $\mathrm{N}_{2}$ flux (baseline). 


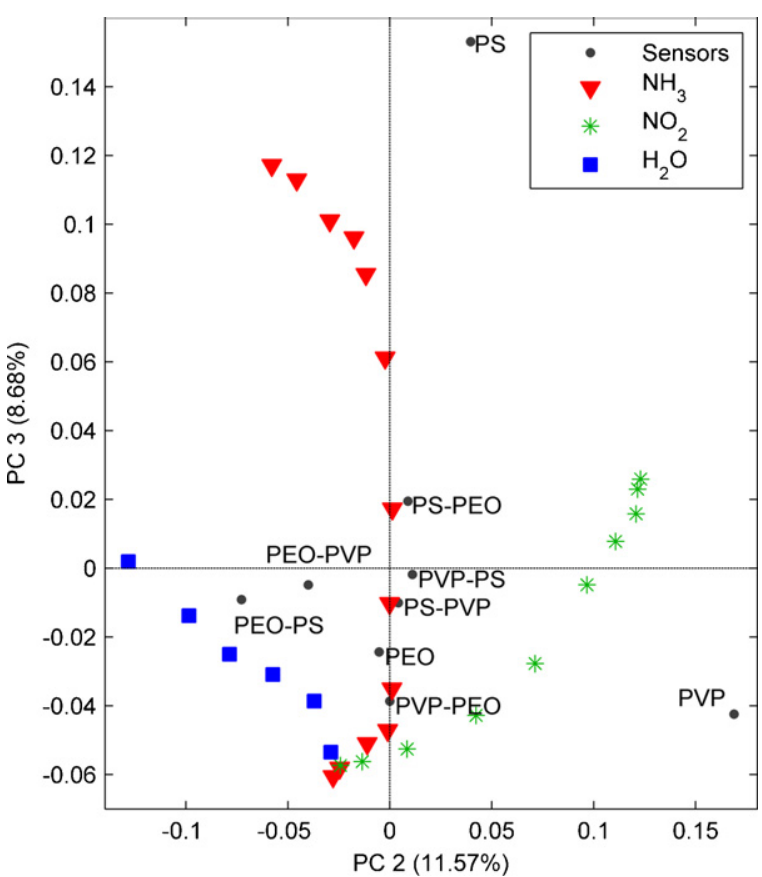

Fig. 4. PCA bi-plot, pointing out the combined loadings and score plot, projected along the second and third principal components. PANi suffix in sensor's name is not reported in the plot.

The extremely reduced swelling effect in PANi-PS (hydrophobic polymer), explains the relatively high responses in current of the sensor to water vapours.

\subsection{Data analysis}

The data analysis performed on the measurement data set were made considering the 9-dimensional space of the sensor array responses to the different concentrations of $\mathrm{NH}_{3}, \mathrm{NO}_{2}$ and water vapours. The first kind of analysis performed was PCA. The scores plot of the first three principal components reported the cumulative percentage variance captured by the PCA model over these 3 PCs and corresponded to $99.25 \%$ of the total. Then such a sensor array might be able to confine the measurements of different analytes in portions of the space of the 3 PCs appearing about orthogonal. The measurements made on different $\mathrm{RH} \%$ showed their maximum variation along $\mathrm{PC} 1$, the $\mathrm{NH}_{3}$ measurements mainly spread along PC3, while the $\mathrm{NO}_{2}$ laid on a direction between PC1 and PC2 (data not shown). A mathematical interpretation of sensors contribution in the differences of the sample scores is presented in the two-dimensional bi-plot in Fig. 4, where differences among the detected analytes are better evidenced by PC2 and PC3 projections. PANi-PS loads mainly along PC3, while PVP loads mainly along the second one. PANi-PEO shows no contribution along PC2 but it is anti-correlated to PANi-PS, indicating that the two sensors give opposite contributions to increasing values of $\mathrm{NH}_{3}$.

The six sensors coated by double fibre layers are generally different from the single layer sensors. For example it is possible to point out that PANi-PVP/PANi-PEO shows the same correlation with ammonia as PANI-PVP (same load over the PC3 direction) but also the same null contribution along PC2 as PANi-PEO. Moreover the order of the deposition of the two fibrous layers seems to give a different behaviour to the final sensor: for example, looking at the PANi-PVP/PANi-PEO vs. PANi-PEO/PANi-PVP and PANi-PS/PANiPEO vs. PANi-PEO/PANi-PS, it is possible to point out how each sensor is approximately orthogonal to its complementary, and how each sensor loads mainly into only one of the two represented principal components. This is also true for the PANi-PVP/PANiPS and PANi-PS/PANi-PVP pair, but only for a small quantity of variance carried by the PC2 and PC3. The PCA analysis suggests that the sensor array is well balanced to separate and discriminate the different classes of analytes under measurement. This capability has been performed by PLS-DA too. In this case a 3dimensional target space has been built, where the coordinates represent the probability that a sample belongs to the $\mathrm{H}_{2} \mathrm{O}, \mathrm{NO}_{2}$ or $\mathrm{NH}_{3}$ classes. The PLS-DA has been built, trying to fit each sample to its corresponding class. A LOOCV method has been used to evaluate the robustness of the model in the prediction of unknown data exhibiting the full capability in the classification of the $\mathrm{NO}_{2}$ and $\mathrm{NH}_{3}$ samples while giving a $20 \%$ of misclassified $\mathrm{H}_{2} \mathrm{O}$ samples and a roughly $5 \%$ of the samples classified as $\mathrm{H}_{2} \mathrm{O}$ coming from a different class. Because of this result, the sensor array has been evaluated in order to consider its capability of estimating the concentration of an $\mathrm{NH}_{3}$ or $\mathrm{NO}_{2}$ sample. This has been accomplished by a PLS regression technique, again supported by a LOOCV. In Fig. 5(a,b) the measured vs. predicted concentration graphs relative to $\mathrm{NO}_{2}$ and $\mathrm{NH}_{3}$, respectively, are reported, as resulting from the PLS regression model. It is worth noting how the model fits the concentration spread for the two compounds. The relevant statistics concerning the PLS model are reported in the figure too, where RMSEC is the root mean square error for calibration data and RMSECV is the root mean square error for cross validation. The PLS regression technique confirmed that the sensors made of
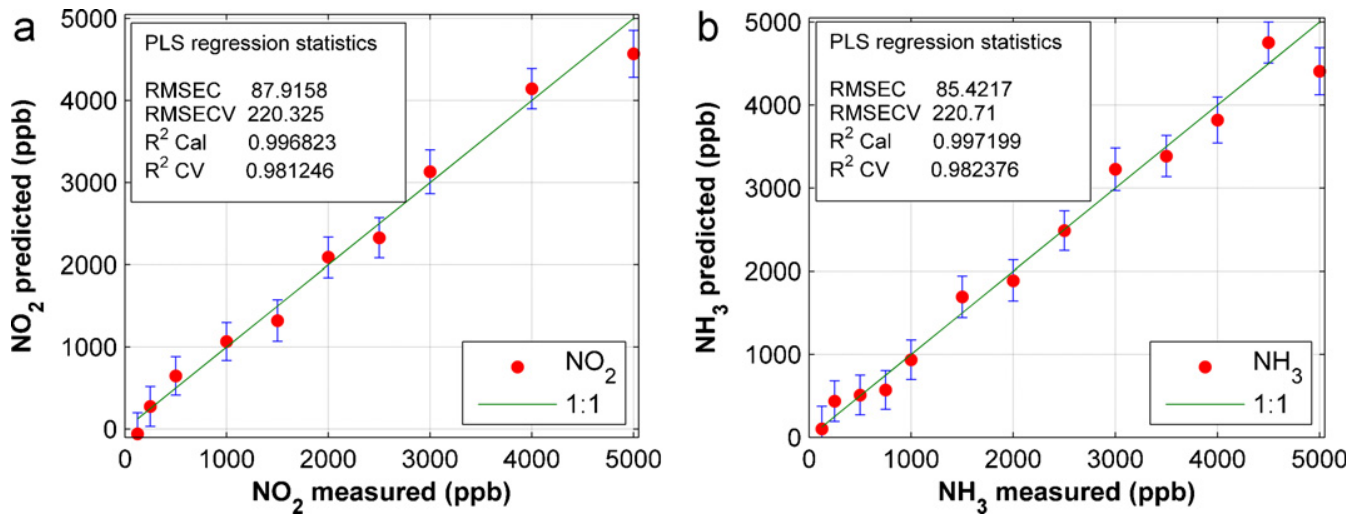

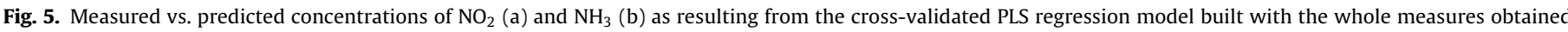

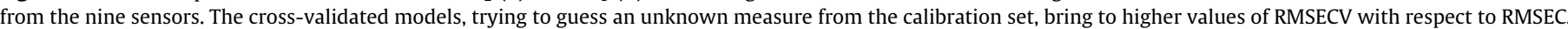
The coefficient of variation reaches its smaller value $(\leq 6 \%)$ at higher concentrations of measured analytes. 
the double fibrous polymeric layer (double-layers) contributed to increase the variability in the sensor array, broadening its sensitivity and creating a wider overlapping selectivity. On the contrary, the regression curves resulting by a PLS regression model of the sensors made by just single polymeric fibres (overlayers) showed a general degradation in the regression capability, in particular for the estimate of the $\mathrm{NH}_{3}$, particularly for low concentration values. In the case of $\mathrm{NO}_{2}$, the model predicted successfully the concentration values up to $1000 \mathrm{ppb}$, while in the case of $\mathrm{NH}_{3}$ the prediction was completely wrong for values up to $500 \mathrm{ppb}$ (in spite of values of about $250 \mathrm{ppb}$ for the case of whole sensor array, both for $\mathrm{NH}_{3}$ and $\mathrm{NO}_{2}$ ). These results indicate that an electronic nose system using only those kind of sensors could reveal suitably an higher limit of recognition, when compared to an equivalent system encompassing also the sensors made of the double layer depositions.

\section{Conclusions}

The present work reported an attempt to join two natural strategies into a bio-inspired artificial system, resulting from the combination of an electronic nose-like device and a nanofibrous artificial epithelium. Thus an array of 9 chemoresistors fabricated on a silicon wafer was coated with different electrospun nanofibres of a suitably doped-PANi blended respectively with three different hosting polymers. Additionally, 3 electrodes were coated with one fibrous polymer (overlays) while the other ones were coated with combinations of 2 polymeric layers (double-overlays). Such enose included a plurality of nanofibres whose electrical parameters were concurrently depending on each polymer exposure to analytes and on the spatial distribution of the interlacing fibres. Further the polymer carriers (PVP, PEO, PS), modifying the typology of the interacting surface (diameter and length of the fibres, roughness, porosity, presence of beads and grains, no woven framework and branched fibres) in addition to the different affinity to the tested analytes, allowed to the interdigitated sensor array getting a wider dynamic range of sensitivities to the gas detected. The electrical parameters of ISA confirmed a Ohmic behaviour (no Schottky barrier). Good performances (like high sensitivities and fast responses) reported to low concentrations of both ammonia and nitrogen dioxide detection were pointed out by response curves. The sensors made of the double fibrous polymeric layer contribute to increase the variability in the sensor array broadening the sensitivity and creating a wider overlapping selectivity. The resulting recognition values for $\mathrm{NH}_{3}$ and $\mathrm{NO}_{2}$ was confirmed to be about $250 \mathrm{ppb}$, in spite of higher values ( $>500 \mathrm{ppb}$ ) when only single fibrous layers (PANiPEO, PANi-PVP and PANi-PS) were used. These preliminary results suggest potential applications of the proposed artificial nose, based on a system of overlaid fibrous polymers, as a potential diagnostic device, able to detect and reveal gases involved in metabolic diseases and released in traces from breath and skin. These sensors also revealed promising features as good stability, low cost, ease to use and operative at room temperature.

\section{Appendix A. Supplementary data}

Supplementary data associated with this article can be found, in the online version, at doi:10.1016/j.bios.2010.10.032.

\section{References}

Albert, K.J., Lewis, N.S., Schauer, C.L., Sotzing, G.A., Stitzel, S.E., Vaid, T.P., Walt, D.R., 2000. Chem. Rev. 100, 2595-2626.

Arshak, K.I., Cunniffe, C., Moore, E.G., Cavanagh, L.M., 2006. IEEE SAS 2006, 30-35.

Aussawasathien, D., Dong, J.H., Dai, L., 2005. Synth. Met. 154, 37-40.

Bai, H., Shi, G., 2007. Sens.-Basel 7, 267-307.

Bathellier, B., Buhl, L.D., Accolla, R., Carleton, A., 2008. Neuron 57, 586-598.

Beccherelli, R., Zampetti, E., Pantalei, S., Bernabei, M., Persaud, K.C., 2010. Sens. Actuators B: Chem. 146, 446-452.

Bishop, A., Gouma, P., 2005. Rev. Adv. Mater. Sci. 10, 209-214.

Canhoto, O., Pinzari, F., Fanelli, C., Magan, N., 2004. Int. Biodeterior. Biodegrad. 54, 303-309.

D’Amico, A., Di Natale, C., Paolesse, R., Macagnano, A., Martinelli, E., Pennazza, G., Santonico, M., Bernabei, M., Roscioni, C., Galluccio, G., Bono, R., Finazzi Agro, E., Rullo, S., 2006. Sens. Actuators B: Chem. 130 (1), 458-465.

De Cesare, F., Pantalei, S., Zampetti, E., Macagnano, A., 2008. Sens. Actuators B: Chem. 131, 63-70.

Di Natale, C., Macagnano, A., Martinelli, E., Paolesse, R., D’Arcangelo, G., Roscioni, C., Finazzi-Agrò, A., D’Amico, A., 2003. Biosens. Bioelectron. 18, 1209-1218.

Gardner, J.W., Bartlett, P.N., 1991. NATO ASI Series E: Applied Sciences, vol. 212. Kluwer Academic Publishing, Dordrecht, The Netherlands.

Goepel, W., 1998. Sens. Actuators B: Chem. 52, 125-142.

Goepel, W., Ziegler, C., Breer, H., Schild, R., Apfelbach, J.J., Malaka, R., 1998. Biosens. Bioelectron. 13, 479-493.

Hao, Q.L., Kulikov, V., Mirsky, V.A., 2003. Sens. Actuators B: Chem. 94, 352-357.

Hierlemann, A., Schweizer-Berberich, M., Weimer, U., Kraus, G., Pfau, A., Gopel, W., 1996. Sens. Update 2,119-180.

Janata, J., Josowicz, M., 2003. Nat. Mater. 2, 19-24.

Kukla, A.L., Shirshov, Y.M., Piletsky, S.A., 1996. Sens. Actuators B: Chem. 37, 135-140.

Li, G.F., Martinez, C., Janata, J., Smith, J.A., Josowicz, M., Semancik, S., 2004. Electrochem. Solid State 7, H44-H47.

Macagnano, A., Zampetti, E., Pantalei, S., Italia, M., Spinella, C., Bearzotti, A., 2009. Olfaction and Electronic Nose: Proceedings of 13th International Symposium, AIP, pp. 373-376.

Macagnano, A., Zampetti, E., Pistillo, B.R., Pantalei, S., Sgreccia, E., Paolesse, R., d'Agostino, A., 2008. Thin Solid Films vol. 516, 7857-7865.

Massart, D.L., Wandeginste, B.G.M., Deming, S.N., Michotte, Y., Kaufman, L., 1998. Chemometrics: A Textbook. Elsevier Science Pub., Amsterdam, The Netherlands, pp. $1-488$

Nef, P., 1998. News Physiol. Sci. 13, 1-5.

Nicolas-Debarnot, D., Poncin-Epaillard, F., 2003. Anal. Chim. Acta 475, 1-15.

Norris, I.D., Shaker, M.M., Ko, F.K., MacDiarmid, A.G., 2000. Synth. Met. 114, 109-114.

Olafsdottir, G., Nesvadba, P., Di Natale, C., Careche, C., Oehlenschläger, J., Tryggvadóttir, S.V., Schubring, R., Kroeger, M., Hei, K., Esaiassen, M., Macagnano, A., Jørgensen, B.M., 2004. Trends Food Sci. Technol. 15, 86-93.

Pavlou, A.K., Magan, N., McNulty, C., Jones, J.M., Sharp, D., Brown, J., Turner, A.P.F., 2002. Biosens. Bioelectron. 17 (10), 893-899.

Pearce, T.C., Schiffman, S.S., Nagle, H.T., Gardner, J.W., 2003. Handbook of Machine Olfaction Electronic Nose Technology. Wiley/VCH Verlag GmbH \& Co. KGaA, Weinheim, p. 592.

Persaud, K.C., Dodd, G.H., 1982. Nature 299, 352-354.

Reneker, D., Chun, I., 1996. Nanotechnology 7, 216-223.

Satir, P., Christensen, S.T., 2007. Ann. Rev. Physiol. 69, 377-400.

Turner, C., Parekh, B., Walton, C., Spanel, P., Smith, D., Evans, M., 2008. Rapid Commun. Mass Spectrom. 22, 526-532.

Tzong-Zeng, W., 1999. Biosens. Bioelectron. 14 (1), 9-18.

Virji, S., Huang, J., Kaner, R.B., Weiller, B.H., 2004. Nano. Lett. 4, 491-496.

Wang, Y., Wang, B., Wang, G., Yin, T., Yu, Q., 2009. Polym. Bull. 63, 259-265.

Wolf, S., Tauber, R.N., 2000. Silicon Processing for VLSI Era, vol. 1. Lattice Press, p. 922 . 